\title{
Model analysis and numeric simulation of wireless communication at high speed
}

\author{
Chunrui Zhang ${ }^{1, \text { a }}$ \\ ${ }^{1}$ School of health sciences and applied psychology, Wuhan Sports University, Hebei 430079, China; \\ acaiweishaonian@126.com
}

Keywords: high speed communication, bit error rate, space optical communication.

\begin{abstract}
With advantages like great security and communication quality, optical communication has make good progress. When this technique is developing at staggering speed, people want to it have more high speed, which can propagate the information much quicker. We want to the speed to enhance to several Gb/s. The next generation of speed is about 40Gb per second. This paper will show us the performance of wireless communication model when the speed has enhanced. We discuss the bit error rate versus different system parameters to illustrate whether the system is good for communication. Some important and specific parameters can also be discussed. These works can be useful to help us know the performance of wireless communication at high speed.
\end{abstract}

\section{Introduction}

With advantages like great security and communication quality, optical communication has make good progress [1-2]. Compared with microwave communication, optical communication has better bit error rate, signal noise rate and easier practical system. With the increasing requirement of human being, we want the higher communication speed. As we know, higher speed means that we need less time to communicate the same number of information [3-6]. The next generation of speed that people want to focus on is about $40 \mathrm{~Gb}$ per second [7]. However, when we enhance the communication speed, the practical system will change at the same time. Actually, the performance of system turns to be worse because of high speed. It is from the reason that high speed makes the data instable.

If the speed data has been enhanced to this kind of level on practical system, we use wave modulation to apply communication [8]. At the same time, avalanche photodiode (APD) will be used to enhance the received signal in communication system [9]. When we speak of the communication process, the effect of atmospheric will affect the performance of signal. When it comes to space downlink system of communication, the atmospheric turbulence is an important phenomenon to decrease the signal performance [10-11]. The key part of atmospheric turbulence is scintillation effect. This effect can make the intensity of received light change irregularly. When we combine the factors above, this paper will discuss how to analyze the performance of system at high speed and how to enhance it. In this paper, the performance of space optical communication at the high level of speed $(40 \mathrm{~Gb} / \mathrm{s})$ with consideration of atmospheric turbulence effect is discussed. The BER performance versus some parameter ratios are also discussed further. We believe our analysis will help people understand the practical communication system. Our numeric simulation and analysis can help people to adjust the system with better BER performance.

\section{Theory}

The avalanche photodiode (APD) will be used to increase the communication quality. When it comes to APD parameters, the current and variation of noise are shown as [12]

$$
\begin{aligned}
& a=G \cdot e \cdot\left(K_{s}(I)+K_{b}\right)+I_{d c} T_{s} \\
& \sigma^{2}=(G \cdot e)^{2} \cdot F \cdot\left(K_{s}(I)+K_{b}\right)+\sigma_{T}^{2}
\end{aligned}
$$

where $F$ is the additional noise factor, $\mathrm{G}$ is the photomultiplier gain factor, $K_{b}=\eta I_{b} T_{s} / h v$ is the photon count of the background light, $h$ is the Planck constant, $v$ is the frequency of the signal light, 
$\sigma_{T}^{2}=2 \kappa_{c} T T_{s} / R_{L}$ is the thermal noise, $T_{s}$ is bit time, $1 / T_{s}$ is communication speed, $T$ is the temperature, $\eta$ is quantum efficiency, $R_{L}$ is load resistance, and $I_{b}=\pi D_{r}^{2} I_{B} / 4$ is the background light in the optical communication system, $D_{r}$ is receiving diameter, $K_{s}$ is the photon count.

Under the impact on the APD with the detector noise, the BER performance on BPSK scheme without effect of atmospheric turbulence is [13]

$$
B E R_{n}=\frac{1}{2} \operatorname{erfc}\left(\sqrt{\frac{a^{2}}{2 \sigma^{2}}}\right)
$$

However, when the atmospheric turbulence is considered, the probability density function of the intensity I owns distribution in downlink optical communication, which is [14]

$$
P_{r}=\frac{1}{\sqrt{2 \pi \sigma_{I}^{2}(r, L)}} \frac{1}{I} \exp \left(-\left(\ln \frac{I}{\langle I(0, L)\rangle}+\frac{2 r^{2}}{W^{2}}+\frac{\sigma_{I}^{2}(r, L)}{2}\right)^{2} /\left[2 \sigma_{I}^{2}(r, L)\right]\right)
$$

where $\langle I(0, L)\rangle=\alpha P_{T} D_{r}^{2} / 2 W^{2}$ is the mean intensify, $D_{r}$ is the receiving diameter, $P_{T}$ is the transmission power, $\alpha$ is the energy loss of the link, $W=W_{o}+\theta L / 2$ is the radius of beam at the receiving plane, $W_{o}$ is transmission diameter, $\theta$ is divergence angle, $L=\left(H-h_{0}\right) \sec (\zeta)$ is the length of the laser link. $H$ and $h_{0}$ are heights of the receiver and the emitter, $r$ is the distance between the beam center and receiving point, $\zeta$ is zenith angle, $\sigma_{I}^{2}(r, L)$ is the variance [14].

Thus, considering the noise of APD and effect of atmospheric turbulence, the final BER is [15]

$$
B E R=\int_{0}^{+\infty} B E R_{n} P_{r} d I
$$

\section{Simulations}

Numeric simulations are based on the following parameters: $\lambda=1550 \mathrm{~nm}$, quantum efficiency of APD $\eta=0.75$, detector amplification $G=100$, duration time $T_{s}=1 / 40 \mu \mathrm{s}$, transmission power $P_{t}=10 \mathrm{~W}$, additional noise factor $F=G^{1 / 2}$, divergence angle $\theta=30 \mu \mathrm{rad}$, zenith angle $\zeta=0, \alpha=1, T=300 \mathrm{~K}$, receiving diameter $D_{\mathrm{r}}=0.5 \mathrm{~m}$, transmission diameter $W_{o}=0.1 \mathrm{~m}$, loading resistor $R_{L}=50 \Omega, h_{0}=100 \mathrm{~m}$, $H=38000 \mathrm{~km}$, bandwidth $B_{\mathrm{w}}=10 \mathrm{~nm}$, dark current $I_{d c}=1 \mathrm{nA}$.

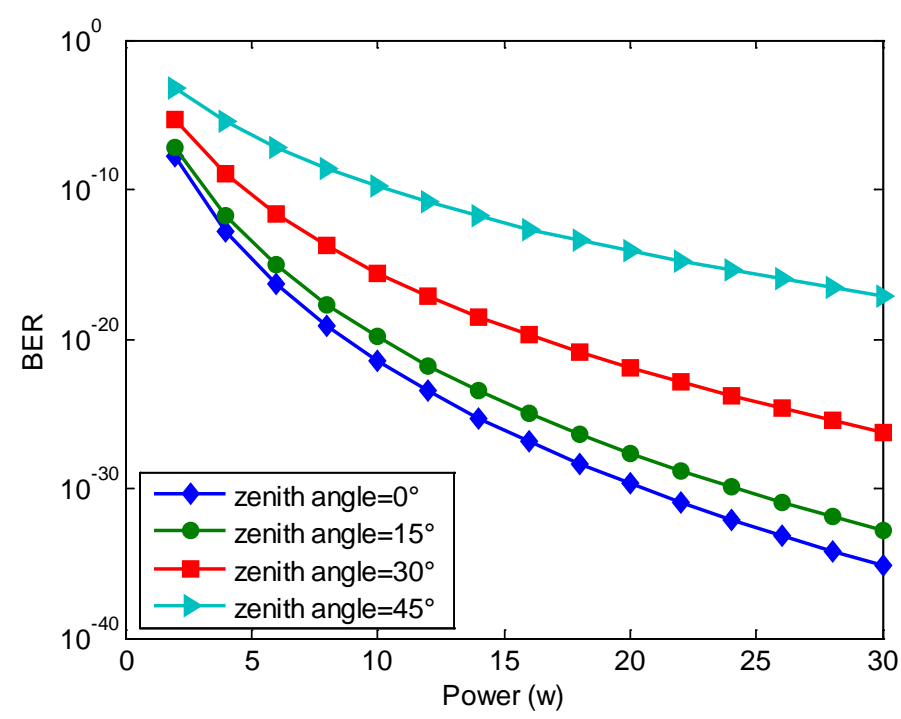

Figure 1: BER versus transmission power

The evolution of BER power is shown in figure. In the region of the power (w) higher 10, the BER is lower than $10^{-10}$ when the zenith angle was $45^{\circ}$. Changing the zenith angle to $0^{\circ}$, the BER decrease 10 orders of magnitude. After the point of 10 power(w), the BER rate of change increase, so does the gap of BER among situation with different doses. Obviously, the BER of system is more sensitive to 
radiation of higher dose. It indicates that we can assure that BER will be at $10^{-30}$ even the zenith angle reaches $15^{\circ}$ if we make the power (w) lower than $30 \mu \mathrm{rad}$.

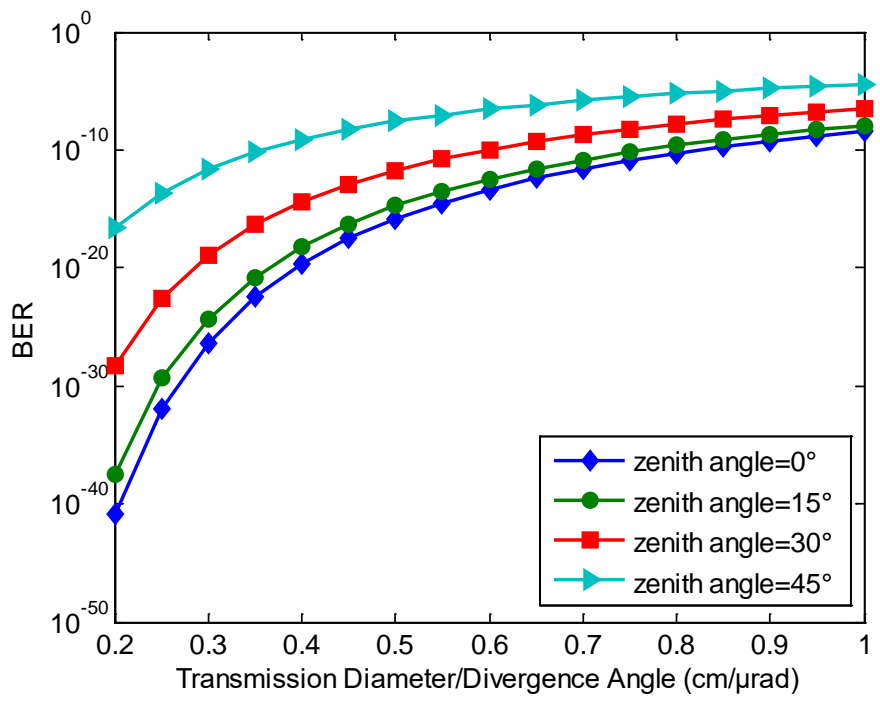

Figure 2: BER versus ratio of transmission diameter and divergence angle

The evolution of BER versus transmit diameter/divergence angle is shown in figure. In the region of small transmit parameter lower than $0.7 \mathrm{~cm}$ the BER is lower than $10^{-10}$ when the zenith angle was $30^{\circ}$. Changing the zenith angle to $45^{\circ}$, the BER increase 10 orders of magnitude. After the point of $0.7 \mathrm{~cm}$, the BER of system is more sensitive to radiation of lower dose. Obviously, the BER of system is more sensitive to radiation of lower dose. It indicates that we can assure that BER will be at most $10^{-10}$ even the divergence angle reach $30^{\circ}$ if we make transmission diameter lower than $0.7 \mathrm{~cm}$.

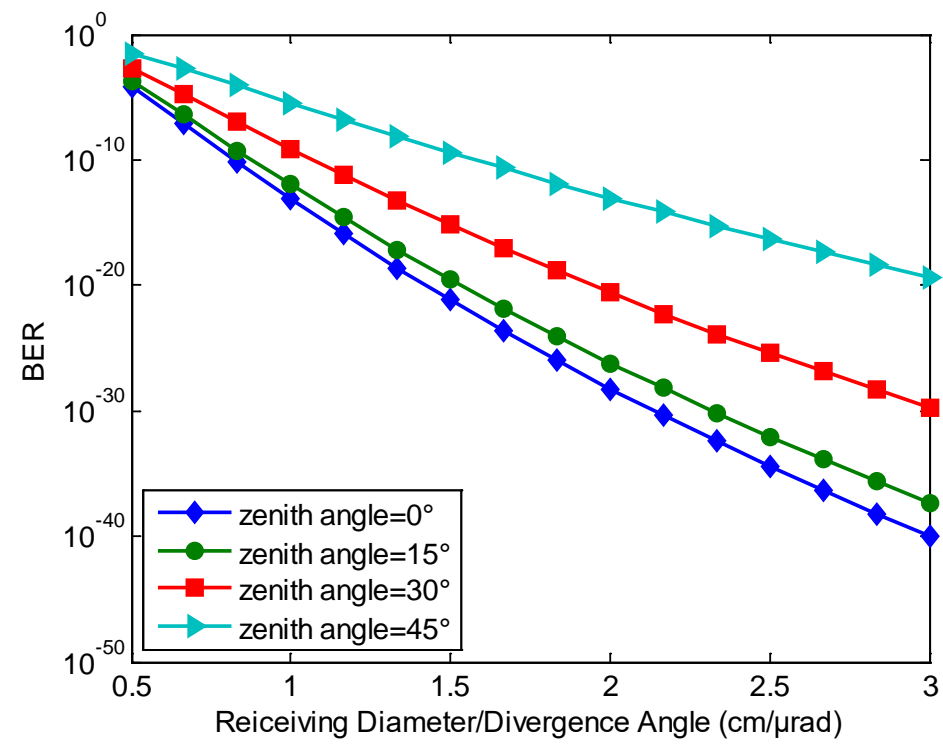

Figure 3: BER versus ratio of receiving diameter and divergence angle

Figure 3 shows that the BER versus receiving diameter/divergence angle. The BER decreases fast when the zenith angle is 0 and 15 degrees. The decreasing speed of 45 degrees is lower than speed of 30 degrees. Obviously, the number of higher degrees, the lower decreasing speed is. Thus, we can adjust the divergence angle in order to exchange the ratio of receiving diameter and divergence angle for the better BER performance. Thus, it is acceptable that it can definitely reduce the expense of system design.

Figure 4 shows the BER versus gain factor/divergence angle. The BER is decreasing fast before the gain factor is $6.5 \mu \mathrm{rad}$. After the point of $6.5 \mu \mathrm{rad}$, the BER starts smoothly increase. The BER decreasing speed is the same. Obviously, the BER is more sensitive to gain factor $6.5 \mu \mathrm{rad}$. It indicates that we can assure the BER will be increased after $6.5 \mu \mathrm{rad}$. This result is helpful for the 
design of system because when the gain factor is decided, they can adjust the angle to get the optimum ratio for the great communication quality.

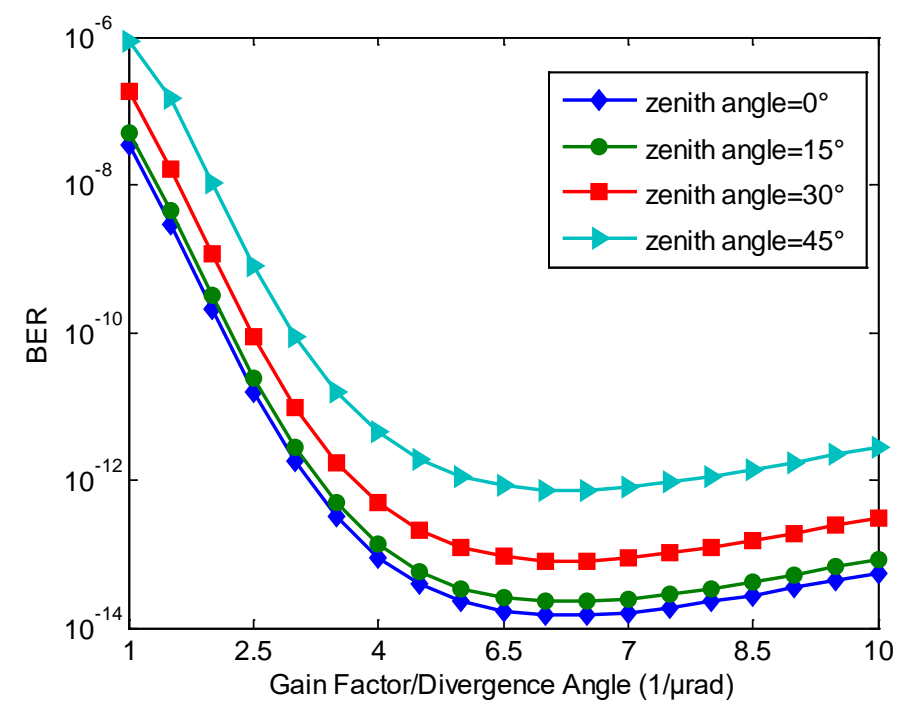

Figure 4: BER versus ratio of gain factor and divergence angle

\section{Conclusions}

In conclusion, the performance of space optical communication at the high level of speed (40Gb/s) with consideration of atmospheric turbulence effect is discussed. The BER performance versus some parameter ratios are also discussed further. From simulation results, large zenith angle will increase the value of BER performance in practical system. We can also see that the power would be better than $10 \mathrm{~W}$ so that we have relatively good BER performance. We can use Er-Yb co-doped amplifier to enlarge the signal in practical system. Apart from it, the ratio of transmission diameter and divergence angle needs to be under 0.4. we can adjust the divergence angle to better position to get better communication quality from simulation results. When we are speaking the APD gain factor, we have the minimum point to have best BER performance. These works can be useful to help us know the performance of wireless communication at high speed.

\section{References}

[1] C. C. Wei, W. Astar, J. Chen, Y. J. Chen, and G. M. Carter, Theoretical investigation of polarization insensitive data format conversion of RZ-OOK to RZ-BPSK in a nonlinear birefringent fiber, Opt. Express 17 (2009) 4306-4316.

[2] L. Andrews, R. Phillips, C. Hopen, Laser beam scintillation with applications, New York, SPIE Press, 2001.

[3] S. T. Le, K. J. Blow, V. K. Mezentsev, S. K. Turitsyn, Bit error rate estimation methods for QPSK co-OFDM transmission, J. Lightw. Technol. 32 (2014) 2951-2959.

[4] L. Yang, X. Q. Gao, M. S. Alouini, Performance analysis of relay-assisted all-optical FSO networks over the strong atmospheric turbulence channels with pointing errors, J. Lightw. Technol. 32 (2014) 4011-4018.

[5] J. Ma, Y. J. Jiang, L. Y. Tan, S. Y. Yu, W. H. Du, Influence of beam wander on bit-error rate in a ground-to-satellite laser uplink communication system, Opt. Lett. 33 (2008) 2611-2613.

[6] S. Y. Lin, Y. C. Chi, Y. C. Su, Y. C. Li, G. R. Lin, An injection-locked weak resonant cavity laser diode for beyond bandwidth encoded 10-Gb/s OOK transmission, IEEE Photonic. J. 7 (2015) 1109-1112. 
[7] X. R. Ma, Y. J. Xu, X. Wang, Z. C. Ding, A novel high precision adaptive equalizer in digital coherent optical receiver, Opt. Commun. 351 (2015) 63-65.

[8] Ivan. B. Djordjevic, Mihajlo. C. Stefanovic, Performance of optical heterodyne PSK systems with Costas loop in multichannel environment for nonlinear second-order PLL model, J. Lightw. Technol. 17 (1999) 2470-2480.

[9] K. Masa, M. Akira, Decision-Directed Costas loop stable homodyne detection for 10Gb/s BPSK signal transmission, IEEE Photon. Technol. Lett. 26 (2014) 319-323.

[10] J. F. Campbell, B. Lin, A. R. Nehrir, F. W. Harrison, M. D. Obland, Binary phase shift keying on orthogonal carriers for multichannel $\mathrm{CO}_{2}$ absorption measurements in presence of thin clouds, Opt. Express 22 (2014) A1634-A1640.

[11] Z. Shu, C. C. John, A SSP-Based control method for a nonlinear Mach-Zehnder interferometer DPSK regenerator, J. Lightw. Technol. 33 (2015) 3788-3795.

[12] Jones, J. Jay, Modern Communication Principle with Application to Digital Signaling, New York, McGraw Hill, 1967.

[13] Y. C. Chi, G. R. Lin, Self optical pulsation based RZ-BPSK and reused RZ-OOK bi-directional oc-768 transmission, J. Lightw. Technol. 32 (2014) 372-83734.

[14] R. M. Gagliardi, S. Karp, Optical telecommunications, Publishing House of Electronics Industry, 1998.

[15] A. Papoulis, S. U. Pillai, Probability random variables and stochastic processes, New York, Mc GrawHill, 1984. 